\title{
Functional characterization and substrate specificity of a novel gene encoding zinc finger-like protein, ZfLp, in Xenopus laevis oocytes
}

\author{
Yasuna Kobayashi', Takahiro Umemoto',2, Yurie Takeshita', Noriko Kohyama1, \\ Masayuki Ohbayashi', Yutaka Sanada² and Toshinori Yamamoto' ${ }^{1}$ \\ ${ }^{1}$ Department of Pharmacotherapy, Division of Clinical Pharmacy, School of Pharmacy, Showa University, \\ 1-5-8 Hatanodai, Shinagawa-ku, Tokyo 142-8555, Japan \\ ${ }^{2}$ Department of Surgery, School of Medicine, Showa University Fujigaoka Hospital, \\ 1-30 Fujigaoka, Aoba-ku, Yokohama-shi, Kanagawa 227-8501, Japan
}

(Received February 4, 2012; Accepted April 17, 2012)

\begin{abstract}
In the present study, we isolated and determined the pharmacological characteristics of a novel gene encoding the zinc finger-like protein (ZfLp). The isolated cDNA consisted of 1,581 base pairs that encoded a 526-amino acid protein. The amino acid sequence of ZfLp is $96 \%$ identical to that of zinc finger protein 415 isoform 5 (ZNF415-5). Reverse-transcription (RT)-polymerase chain reaction (PCR) analysis revealed that the ZfLp mRNA is expressed in the breast, lung, stomach, small intestine colon and ovary, but not in the liver. When expressed in Xenopus laevis oocytes, ZfLp mediated the high affinity transport of $\left[{ }^{3} \mathrm{H}\right]$ paclitaxel (taxol) in a sodium-independent manner $\left(K_{\mathrm{m}}=336.7 \pm 190.0 \mathrm{nM}\right)$. The uptake of $\left.{ }^{3} \mathrm{H}\right]$ paclitaxel (taxol) by ZfLp was trans-stimulated by glutarate and glutathione (GSH). A cis-inhibition experiment revealed that ZfLp-mediated transport of $\left[{ }^{3} \mathrm{H}\right]$ paclitaxel (taxol) is inhibited by several organic solutes specifically clotrimazole. Using several clotrimazole derivatives, we found that N-benzylimidazole would be a minimum unit for producing the inhibition of ZfLp-mediated drug uptake. Our results may provide insights into the novel role of soluble protein, such as ZNF, in the human body. Our results, therefore, would be expected to facilitate research on the novel role of ZNFs and on the discovery of novel drugs for targeting ZNF-related proteins such as ZfLp.
\end{abstract}

Key words: Zinc finger-like protein, Drug transporter, Organic solutes, Toxicity

\section{INTRODUCTION}

The zinc finger proteins (ZNFs) are a single class of transcription factors in the human genome. ZNFs constitute the largest individual family of nucleic acid-binding proteins (Looman et al., 2002). A zinc finger has a conserved motif of 28 amino acids, which is often repeated within a protein and may be involved in protein-protein or DNA-protein interactions (Klug and Rhodes, 1987; $\mathrm{Wu}, 2002$; Lupo et al., 2011). ZNFs can coordinate one or more zinc ions to stabilize their folds. In addition, these proteins are structurally diverse and exist among proteins that perform a broad range of functions in various cellular processes such as repair, replication, translation, transcription, metabolism, signaling, cell proliferation and apoptosis (Mitchell and Tjian, 1989). Thus, ZNFs play central roles in regulating the expressions of a variety of genes.

Drug concentration in the blood and the target organs regulate several sequential steps such as transport, intracellular processing, elimination, excretion, and metabolism. Although these processes have been studied in the liver, kidney, brain, small intestine and placenta, the uptake step is an important process because many different kinds of drug carrier proteins are known to regulate the drug concentrations in these organs (Saier, 2000; Van Aubel et al., 2000; Leonard et al., 2003; Leonessa and Clarke, 2003; Rizwan and Burckhardt, 2007). Several different kinds of xenobiotic transporters have been isolated and well characterized. To date, 51 solute carrier (SLC) families have been classified (http://www.bioparadigms. org/slc/intro.htm). Among them, the SLC21A and $22 \mathrm{~A}$ families are considered to be most important molecules

Correspondence: Toshinori Yamamoto (E-mail: yamagen@pharm.showa-u.ac.jp) 
for drug clearance from the body. For example, LST-1/ OATP1B1[SLCO1B1] transports statins (Simonson et al., 2004; Kameyama et al., 2005), ezetimibe glucronide (Nozawa et al., 2005; Oswald et al., 2008), SN-38 (Nozawa et al., 2005), and valsartan (Maeda et al., 2006). Human organic anion transporter 2 (hOAT2[SLC22A7]), 5 (hOAT5[SLC22A10]) and 7 (hOAT7[SLC22A9]) are also considered to be a key molecule in hepatic handling of organic anions since this protein mediates the transport of methotrexate, theophylline, erythromycin, prostaglandin $\mathrm{E}_{2}\left(\mathrm{PGE}_{2}\right)$, cAMP, $\alpha$-ketoglutarate, azidodeoxythymidine (AZT), tetracycline and $p$-aminohippurate (PAH) (Sun et al., 2001; Babu et al., 2002; Takeda et al., 2002; Kobayashi et al., 2005a; Shin et al., 2007).

With regard to the secondary structure of these transporters, many investigators have predicted there are 10-12 transmembrane domains (TMDs) (Sekine et al., 1997; Sweet et al., 1997; Cha et al., 2000, 2001; Hagenbuch and Meier, 2004; Koepsell and Endou, 2004). Likewise, the ATP-binding cassette (ABC) transporter family, such as breast cancer resistance protein (BCRP $[A B C G 2])$, is predicted to have 6 TMDs (Leslie et al., 2005). In addition, we have proposed that human organic solute carrier partner 1 (hOSCP1, formally named organic solute carrier protein 1) is predicted to have 3 TMDs (Kobayashi et al., 2005b). The secondary structure model of 4F2hc is predicted to have a single transmembrane domain (Wells et al., 1992; Kanai et al., 1998). Similarly, related to $\mathrm{b}^{0,+}$ amino acid transporter (rBAT), the lambda light chain of human immunoglobulin surface antigen-related gene (IgLC-rG), and ribosomal protein L3 (RPL3) are predicted to have one and two TMDs, respectively (Bertran et al., 1992a, 1992b; Wells et al., 1992; Lee et al., 1993; Kanai et al., 1998; Kobayashi et al., 2005b, 2010a and 2010b). Thus, the TMD signature seems to be an important structural signature(s) for activating drug transport.

In the present study, we examined the transport activity of a novel gene encoding zinc finger-like protein (ZfLp) using a $X$. oocyte expression system. Although ZNFs plays an important role in regulating the expression of many kinds of genes, our results indicate that isolated ZfLp may function as a drug carrier protein. Our findings would provide new insights into a novel function of ZNF, especially a ZNF-related gene such as ZfLp.

\section{MATERIALS AND METHODS}

\section{Materials}

$\left[{ }^{3} \mathrm{H}\right]$ Paclitaxel (taxol) $(20 \mathrm{Ci} / \mathrm{mmol})$ was purchased from American Radiolabeled Chemicals, Inc. (St. Louis, MO, USA). [ $\left.{ }^{3} \mathrm{H}\right] 5$-Fluorouracil (5-FU) (13 Ci/mmol) was purchased from Moravek Biochemicals, Inc. (Brea, CA, USA). Deoxycytidine $\left[5^{\prime}-\alpha-{ }^{32} \mathrm{P}\right]$ triphosphate (dCTP) (111 TBq/mmol) was obtained from Muromachi Yakuhin Kaisha, LTD (Tokyo, Japan). N-Triphenylimidazole, N-diphenylmethylimidazole, and N-phenylpropylimidazole were synthesized as previously described (Kobayashi et al., 1993). N-Benzylimidazole and clotrimazole were purchased from Sigma-Aldrich, Co. (St. Louis, MO, USA). All other chemicals not listed here were of the highest grade commercially available.

\section{Construction of cDNA library and isolation of ZfLp}

A nondirectional cDNA library was prepared from human breast poly (A) ${ }^{+}$RNA using the Superscript Choice System (Life Technologies, Gaithersburg, MD, USA) and was ligated into a phage vector $\lambda$ ZipLox EcoRI arms (Life Technologies). Human breast poly (A)+ RNA was purchased from BD Biosciences Clontech (Palo Alto, CA, USA).

An Expressed Sequence Tag (EST) data base search for "Query SLCO2A1" (GenBank accession number NM_005630) and an EST clone (BU944345) were identified. After PCR amplification of this EST clone (left primer, 5'-ATACGGACAGACTGGGATGC-3'; right primer, 5'-GAGGTGGCTTCCAGTACAGC-3'), the PCR product was labeled with $\left[5^{\prime}-\alpha-{ }^{32} \mathrm{P}\right] \mathrm{dCTP}$ by random priming ( ${ }^{\mathrm{T} 7}$ Quick Prime Kit, Amersham Pharmacia Biotech), and the library was screened with an EST clone as a probe under low stringency conditions. Replicate filters of a phage library were hybridized overnight in a hybridization solution (50\% formamide; $5 \mathrm{x}$ standard saline citrate (SSC); $3 \mathrm{x}$ Denhardt's solution; $0.2 \%$ sodium dodecy sulfate (SDS); $10 \%$ dextran sulfate; $0.3 \mu \mathrm{g} / \mathrm{ml}$ denatured salmon sperm DNA; $2.5 \mathrm{mM}$ sodium pyrophosphate; $25 \mathrm{mM}$ 4-morpholineethane sulfonic acid (MES); $0.03 \%$ Antifoam A; $\mathrm{pH} 6.5$ ) at $37^{\circ} \mathrm{C}$ overnight. The filters were washed in $3 \mathrm{x}$ SSC and $0.5 \%$ SDS at $37^{\circ} \mathrm{C}$. cDNA inserts in positive $\lambda$ ZipLox phages were recovered in a plasmid pZL1 vector by in vitro excision.

\section{cDNA sequence}

Double-stranded cDNA of isolated clones were sequenced in both directions. Deleted clones, obtained by a KiloSequence deletion kit (Takara, Tokyo, Japan), 
Isolation and pharmacological characterization of a novel gene, ZfLp

and specifically synthesized oligonucleotide primers were used for sequencing ZfLp cDNA, which was sequenced by the dye terminator method using a dye primer cycle sequencing kit (ver. 3.1., Applied Biosystems, Foster City, CA, USA) and automated Applied Biosystems 310 DNA sequencer. The sequence and the phylogenic tree were analyzed using DNASIS ${ }^{\circledR}$-Pro. Ver. 2.02 (HITACHI Software Engineering, Yokohama, Japan).

\section{cRNA synthesis and functional characterization of ZfLp in Xenopus laevis oocytes}

Selection and isolation of stage V and VI defolliculated $X$. oocytes was performed as previously described (Kobayashi et al., 2010a, 2010b). Collagenase A (Boehringer Mannheim, Mannheim, Germany) was used to remove the follicular layer at a final concentration of 2.0 $\mathrm{mg} / \mathrm{ml}$ in a oocyte Ringer 2 (OR2) solution ( $83 \mathrm{mM} \mathrm{NaCl}$, $2 \mathrm{mM} \mathrm{KCl}, 1 \mathrm{mM} \mathrm{MgCl}$, 5 mM 2-[4-(2-hydroxyethyl)1-piperazinyl]ethanesulfonic acid (HEPES), pH 7.5). The ZfLp cDNA was linearized with Kpn1, and capped cRNA was transcribed in vitro by Sp6 RNA polymerase. Defolliculated oocytes were microinjected with $50 \mathrm{ng}$ of in vitro transcribed cRNA and incubated for two days in a modified Barth's solution containing gentamicin $(50 \mu \mathrm{g} / \mathrm{ml})$ at $18-20^{\circ} \mathrm{C}$. Uptake experiments of radiolabeled substrates, as indicated in each experiment, were performed in ND96 solution (96 mM NaCl, $2 \mathrm{mM} \mathrm{KCl,} 1.8 \mathrm{mM} \mathrm{CaCl}_{2}$, $1 \mathrm{mM} \mathrm{MgCl}_{2}, 5 \mathrm{mM}$ HEPES, $\mathrm{pH}$ 7.4) at room temperature. Oocytes were incubated in $150 \mu \mathrm{l}$ of the same solution containing radiolabeled substrates. For $\mathrm{Na}^{+}-$ dependent transport experiments, we used ND96 buffer containing lithium chloride, choline chrolide, and mannitol with an equivalent amount of sodium chloride. Concentration-dependent uptake experiments of $\left[{ }^{3} \mathrm{H}\right]$ paclitaxel (taxol) in oocytes expressing ZfLp were performed at final concentrations of 1, 10, 20, 50, 100, 200 and $400 \mathrm{nM}$. Time-dependent transport of $\left[{ }^{3} \mathrm{H}\right]$ paclitaxel (taxol) via ZfLp were performed at 15, 30, 60, 120 and $150 \mathrm{~min}$. The compound was incubated with expressing ZfLp oocytes for $1 \mathrm{hr}$ at room temperature, stopped with ice-cold ND96 solution and washed 5 times. Three oocytes were transferred to one scintillation vial and dissolved in $200 \mu 1 \quad 10 \%$ SDS. A scintillation cocktail was added, and radioactivity was counted. Counts in cRNA minus uninjected oocytes were subtracted from the counts in ZfLp cRNA-injected oocytes. Data are presented as mean \pm S.E., except for kinetic constants for which the error represents the error of the fit. $K_{\mathrm{m}}$ indicates the Michaelis-Menten constant (nM). The experiments were repeated with oocytes from at least five to six frogs.

\section{RT-PCR analysis}

ZfLp cDNA was amplified by RT-PCR using the sense primer 5'-GACCAACGCGATAGAAGAGG-3' and antisense primer 5'-ATTCAAGGCTTTGTCGCACT-3' to yield a 312 fragment. RT-PCR was performed under the following conditions: 1 cycle at $60^{\circ} \mathrm{C}$ for $30 \mathrm{~min}, 1$ cycle at $94^{\circ} \mathrm{C}$ for $2 \mathrm{~min}, 35$ cycles at $94^{\circ} \mathrm{C}$ for $1 \mathrm{~min}$, annealing at $53^{\circ} \mathrm{C}$ for $1.5 \mathrm{~min}$ followed by a final extension at $49^{\circ} \mathrm{C}$ for $10 \mathrm{~min}$ according to the manufacturer's instructions (BD Biosciences Clontech, Palo Alto, CA, USA).

\section{Quantification of ZfLp mRNA expression}

Total RNAs from 7 human tissues (breast, lung, liver, stomach, small intestine, colon, and ovary) were purchased from BioChain Institute, Inc. (San Leandro, CA, USA). RNAs were reverse-transcribed and realtime PCR was operated on a realplex ${ }^{2}$ system (eppendorf Japan, Tokyo, Japan) using the TaqMan Gene Expression Assay for ZNF415 (Applied Biosystems, Rotkreuz, Switerland). Constitutively expressed human glyceraldehyde-3-phosphoate dehydrogenase (G-3-PDH) was measured as an internal control for sample normalization (Applied Biosystems).

\section{cis-Inhibition study}

For inhibition experiments, oocytes expressing ZfLp were incubated for $1 \mathrm{hr}$ in ND96 solution containing $100 \mathrm{nM}\left[{ }^{3} \mathrm{H}\right]$ paclitaxel (taxol) in the presence or absence of inhibitors at a final concentration of $10 \mu \mathrm{M}$. Cyclosporine A, miconazole, clotrimazole, troleandomycin, azithromycin, erythromycin, N-triphenylimidazole, N-diphenylmethylimidazole, N-benzylimidazole, and N-phenylpropylimidazole were directly dissolved in ND96 solution from the stock solution. These inhibitors were dissolved in dimethyl sulfoxide (DMSO) and diluted to a final concentration as described above. The final concentration of DMSO in the assay medium did not exceed $<1 \%$.

\section{Statistical analysis}

Kinetic data from experiments measuring the uptake of radiolabeled substrates were fit to the Michaelis-Menten equation by nonlinear least-squares regression analysis with standard errors derived from these curves (GraphPad Prism Version 4.01). Comparisons of data measuring initial rates of uptake of radiolabeled substrates in the presence and absence of inhibitors were performed by oneway ANOVA (GraphPad Prism Version 4.01), or unpaired Student's $t$ test. 


\section{RESULTS}

Because the SLC2A1/SLCO2A1/OATP2A1 (NM 005630) gene is expressed ubiquitously in the human body (http://www.bioparadigms.org/slc/menu. asp), we assumed that this gene is a good tool for isolating a novel gene. After multiple rounds of screening using $\left[{ }^{32} \mathrm{P}\right] \mathrm{dCTP}-$ labeled EST clone (BU944345) under low stringency condition, we isolated several clones. Two clones had overlapping identical sequences, identical to ZfLp; the other clones had approximately $80 \%$ similarity with Ras and Rab interactor 2 (CAI19340), ras inhibitor (AAA36553), hypothetical protein (CAB66858), Ras and Rab interactor 2, isoform CRA_b (EAX10217), unnamed protein product (BAG52947) at the amino acid level. The deduced amino acid sequence of ZfLp exhibited $<10 \%$ identical to that of the SLC2A1/SLCO2A1/OAT$P 2 A 1$. The nucleotide and deduced amino acid sequences of ZfLp are shown in Fig. 1A. ZfLp cDNA consist of 1,581 nucleotides and codes for 526 amino acids with a calculated molecular mass of $60.4 \mathrm{kDa}$. There are four consensus sequences for $\mathrm{N}$-glycosylation sites $\left(\mathrm{Asn}^{88}\right.$, $\left.\mathrm{Asn}^{103}, \mathrm{Asn}^{4258}, \mathrm{Asn}^{481}\right)$, fourteen PKC sites $\left(\mathrm{Thr}^{90}, \mathrm{Ser}^{105}\right.$, $\mathrm{Thr}^{155}, \mathrm{Thr}^{90}, \mathrm{Ser}^{161}, \mathrm{Thr}^{203}, \mathrm{Ser}^{225}, \mathrm{Thr}^{300}, \mathrm{Ser}^{309}, \mathrm{Ser}^{322}$, $\mathrm{Ser}^{400}, \mathrm{Ser}^{477}, \mathrm{Thr}^{490}, \mathrm{Ser}^{505}$ ), nine zinc finger $\mathrm{C}_{2} \mathrm{H}_{2}$ sites $\left(\mathrm{Cys}^{217}, \mathrm{Cys}^{245}, \mathrm{Cys}^{273}, \mathrm{Cys}^{329}, \mathrm{Cys}^{357}, \mathrm{Cys}^{385}, \mathrm{Cys}^{441}\right.$, $\mathrm{Cys}^{469}, \mathrm{Cys}^{497}$ ). Rose hydropathy analysis (Window 11) of ZfLp protein revealed that this protein has several TMDs (data not shown). However, Kyte and Doolittle (Window 11), TMpred (http://www.ch.embnet.org/software/TMPRED_form.html), Janin (Window 11) and SOSUI (http://bp.nuap.nagoya-u.ac.jp/sosui/sosui submit.html) hydropathy analyses predicted that ZfLp protein has no hydrophobic stretches long enough to span the membrane (data not shown).

When we submitted the ZfLp cDNA sequence to Genbank, a similar sequence from humans had been deposited to GenBank (GenBank ${ }^{\mathrm{TM}} / \mathrm{EMBL} / \mathrm{DDBJ}$ : BAA92059, unnamed protein product, Homo sapiens). The amino acid sequence of ZfLp is $96 \%$ identical to that of ZNP415-5. We also identified several similar human gene products such as EAW72118, ABI51311, EAW72117, EAW72115, NP 060825, NP 001129510, ABI51312, ABI51310, EĀW72116, ĀAH63880, BAG53073, XP_541449, NP_001166145, AAK37403, NP 115973, BAF82683, BAG53339, and BAG37173 that exhibited approximately $87 \%$ and $96 \%$, respectively, predicted amino acid identity. Therefore, we constructed the phylogenic tree using some of these similar sequences in blast program (http://blast.ncbi.nlm.nih.gov/Blast.cgi) and found that $\mathrm{ZfLp}$ is positioned within the human zinc finger protein superfamily (Fig. 1B). These findings lead us to conclude that isolated cDNA is a novel gene that belongs to the human zinc finger protein.

Based on the Rose hydropathy analysis, we hypothesized that ZfLp might have a transport function. ZfLp cRNA-injected Xenopus oocytes were used for the transport characterization. Although ZfLp cRNA-injected oocytes exhibited no transport of several organic solutes such as $\left[{ }^{3} \mathrm{H}\right]$ allopurinol, $\left[{ }^{3} \mathrm{H}\right]$ probenecid, $\left[{ }^{3} \mathrm{H}\right]$ valproate, $\left[{ }^{14} \mathrm{C}\right] p$-aminohippuric acid, $\left[{ }^{14} \mathrm{C}\right]$ tetraethylammonium, $\left[{ }^{14} \mathrm{C}\right]$ theophylline, $\left[{ }^{14} \mathrm{C}\right]$ nicotinamide, $\left[{ }^{14} \mathrm{C}\right] 6$-mercaptopurine, $\left[{ }^{14} \mathrm{C}\right]$ salicylate (data not shown); however, we found that oocyte expressing ZfLp exhibited the transport of $\left[{ }^{3} \mathrm{H}\right] 5-\mathrm{FU}$ and $\left[{ }^{3} \mathrm{H}\right]$ paclitaxel (taxol) to about 2.1 and 4.3 fold that of the control oocytes, respectively (Table 1). Our results indicate that $\left[{ }^{3} \mathrm{H}\right]$ paclitaxel (taxol) and $\left.{ }^{3} \mathrm{H}\right] 5-$ FU would be good substrates for determining the pharmacological characterization of ZfLp.

We subsequently examined the transport characterization of ZfLp using [ $\left.{ }^{3} \mathrm{H}\right]$ paclitaxel (taxol). Firstly, we tested the effect of $\mathrm{pH}$ on the uptake of $\left[{ }^{3} \mathrm{H}\right]$ paclitaxel (taxol) by ZfLp-expressing oocytes. As shown in Fig. 2A, there was a different in $\left[{ }^{3} \mathrm{H}\right]$ paclitaxel (taxol) transport with a $\mathrm{pH}$ at 7.5; the activity was decreased by $\mathrm{pH} 5.5$. Thus, the uptake of $\left[{ }^{3} \mathrm{H}\right]$ paclitaxel (taxol) mediated by ZfLp is sensitive to $\mathrm{pH}$. Based on this finding, we next examined the concentration-dependent transport of $\left[{ }^{3} \mathrm{H}\right]$ paclitaxel (taxol) via ZfLp (Fig. 2B). The ZfLp-mediated uptake of [ $\left.{ }^{3} \mathrm{H}\right]$ paclitaxel (taxol) showed saturable kinetics and could be modeled by the Michaelis-Menten equation. Nonlinear regression analyses yielded $K_{\mathrm{m}}$ values of $336.7 \pm 190.0 \mathrm{nM}$. Time-dependent transport of $\left[{ }^{3} \mathrm{H}\right]$ paclitaxel (taxol) via oocytes expressing ZfLp was subsequently investigated and revealed that the transport activity increased linearly until $15 \mathrm{~min}$ and reached maximum at $60 \mathrm{~min}$ of incubation (Fig. 2C). The effect of extracellular cation on the uptake of $\left[{ }^{3} \mathrm{H}\right]$ paclitaxel (taxol) was studied (Fig. 2D). The uptake of $\left[{ }^{3} \mathrm{H}\right]$ paclitaxel (taxol) via ZfLp was not affected by the replacement of extracellular sodium with lithium, choline, and mannitol, indicating that transport of $\left[{ }^{3} \mathrm{H}\right]$ paclitaxel (taxol) via ZfLp exhibited an $\mathrm{Na}^{+}$-independent carrier protein. We next examined the trans-stimulatory effect of GSH and glutarate to elucidate whether these endogenous compounds trans-stimulate the transport of $\left[{ }^{3} \mathrm{H}\right]$ paclitaxel (taxol) via ZfLp. As shown in Fig. $2 \mathrm{E}$, transport of $\left[{ }^{3} \mathrm{H}\right]$ paclitaxel (taxol) by ZfLp exhibited the trans-stimulatory effect in the presence of both GSH and glutarate.

In order to elucidate the distribution and the localization of ZfLp mRNA in the human body, we chose some human tissues and performed RT-PCR analysis. A band 
Isolation and pharmacological characterization of a novel gene, ZfLp

A

ATG TCC AGG GTC GAT ACGG GCC CIT AAA ATC CCCC GCA CAC TCC ACC CCA GAT CTG TCT CGG AAC TGT GTA ATC AAG GAA GTA GCA CCA GAA CAG GAA GGT AAC CCA GGA GAA GTA TIC CAC ACA GVG ACA TTG GAA GAA CAT GAA AAA CAT GAC ATT GAA GAG TTT TGC TTC AGG GAA ATC AAG AAA AAA ATA CAC GAC TTT GAC TGT CAG TGG AGA GAT GAT GAA AGA AAT TGC AAC AAA

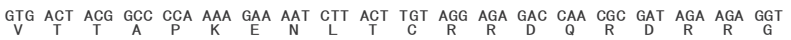
ATA GGA AAC AAG TCT ATT AAA CAT CAG CTT GGA TTA AGC TTT CTA CCA CAT CCC CAT GAA CTG CAG CAG TTT CAA GCT GAA GGG AAA ATT TAT GAA TGT AAC CAT GIT GAGG AAG TCT GTC AAC CAT GGT TCC TCA GIT TCA CCA CCC CAA ATA CTT TCT TCT ACC GTC AAA ACC CAT GIT TCT AAT AAA TAT GGG ACT GAT TTC ATC TGT TCT TSA TTA CTC ACA CAA GAA GAG AAA TCA TGC ATT AGG GAA AAA CCT TAC AGA TAT ATT GAG TGC GAC AAA GCC TTG AAT CAT GGC TCA CAC ATG ACT GTA GGT CAG GTA AGT CAT TCT GGA GAG AAA GGA TAT AAA TGT GAT CTG TGT GGG AAG GTC TIT AGT CAA AAA TSA AAC CIT GGG CGT CAT TGG AGA GIT CAT ACT GGA GAG AAA CCA TAC AAA TGT AAT GAA TGT GAC AGA AGT TTC AGT CGC AAC TCA TGC CTT GCA CTA CAT CGG AGA GTT CAC ACT GGA GAG AAA CCT TAC AAA TGT TAT GAG TGT GAC AaG GTC TTC AGT CGA AAT TCA TGC CTT GCA CTA CAT CAG AAA ACT CAT ATT GGA GAG AAA CCT TAC ACG

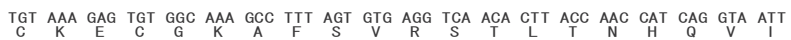
CAT AGT GGC AAG AAA CCT TAC AAA TGC AAT GAA TGT GGC AAGG GVG TTC AGT CAG ACT TCA AGC CIT GCA ACT CAT CAG AGA ATT CAC ACT GGG GAG AAA CCA TAC AAG TGT AAT GAA TGT GGT AAA GTC TTC AGT CAG ACT TCA AGC CTT GCA AGG CAT TGG AGA ATT CAT ACT GGA GAG AAA CCT TAC AAA TGC AAT GAA TGT GGT AAG GTT TTC AGT TAC AAT TCA CAC CLT GCG AGT CAT CGG AGA GIT CAT ACT GGA GAG AAA CCT TAC AAG TGT AAT GAG TGT GGG AAA GCC TTT AGT GIG CAT TCG AAC TTA ACT ACC CAT CAG GTC ATC CAT ACT GGA GAG AAG CCT TAC AAA

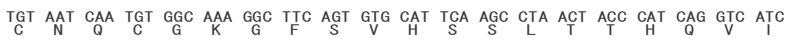

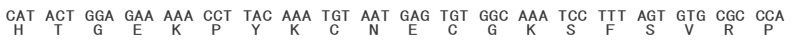
AAC CTC ACT AGA CAT CAG ATA ATC CAT ACT GGA AAG AAA CCT TAC AAA TGT AGT GAT TGT $\underset{G}{G G G}$ AAG TCC TPT AGT GTG CGC CCA AAC CTC TTC AGA CAT CAA ATT ATC CAT ACT AAG GAG 60 120 180 240 300 360 420 480 540 600 660 720 780 840 900 960 1020 1080 1140 1200 1260 1320 1380 1440 $\underset{K}{A A A} \underset{P}{C C T} \underset{Y}{T A T} \underset{K}{\text { AAA }} \underset{\mathrm{R}}{\operatorname{AGA}} \underset{\mathrm{N}}{\text { AAT }} \underset{*}{\text { TAA }} \mathbf{3}^{\prime}$

B

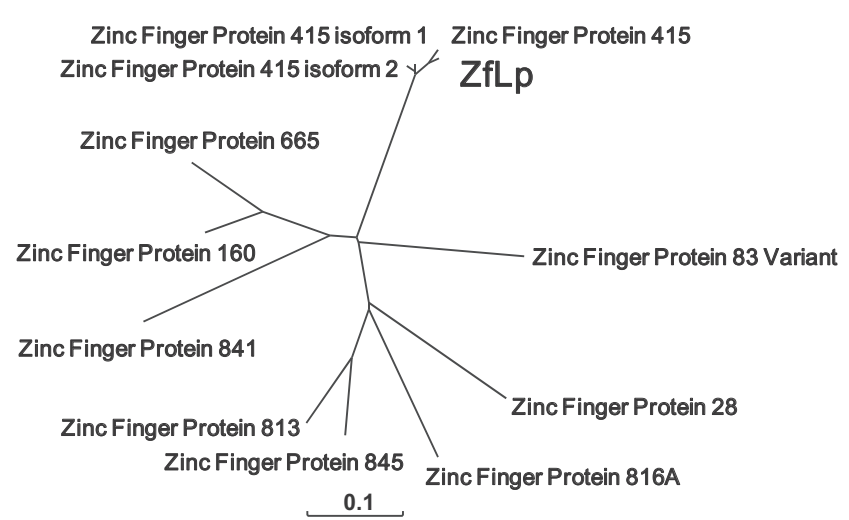

Fig. 1. cDNA and amino acid sequences and phylogenic tree analysis of the zinc finger-like protein (ZfLp). A, cDNA and deduced amino acid sequences of the ZfLp. B, Phylogenetic relationship between zinc finger-like protein and several ZNF-related proteins. The phylogenetic tree was constructed using DNAsis-Pro. Version 2.02 (HITACHI Software Engineering). Branch length is drawn to scale. ZfLp, zinc finger-like protein.

with 312 bp was detected in the breast, lung, stomach, small intestine, colon, and ovary (Fig. 3A). No apparent PCR band was detected in human liver. To elucidate further detailed expression of the ZfLp gene, we subsequent- ly performed real-time PCR analysis. The mRNA expression of this clone exhibited a rank order of the ovary $(\mathrm{Ov})$ $>\operatorname{lung}(\mathrm{Lu})>$ breast $(\mathrm{Br})>\operatorname{stomach}(\mathrm{Sto})>\operatorname{colon}(\mathrm{Col})>$ small intestine $(\mathrm{SI})=$ liver $(\mathrm{Li})($ Fig. $3 \mathrm{~B})$. Thus, we found 
A
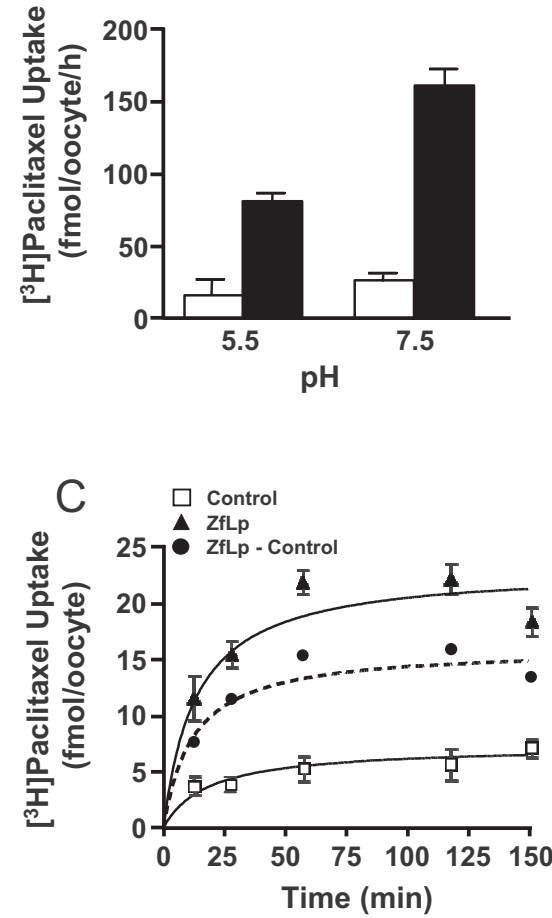

$E$

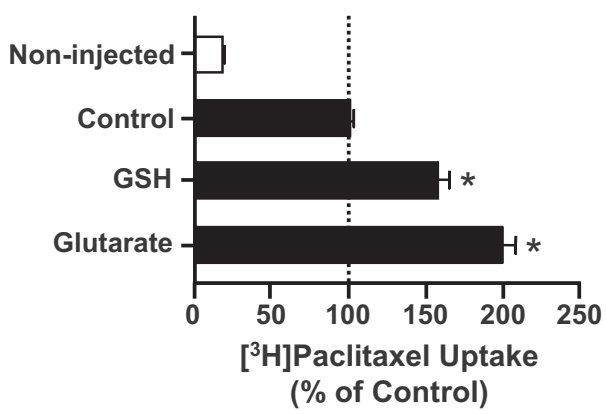

B

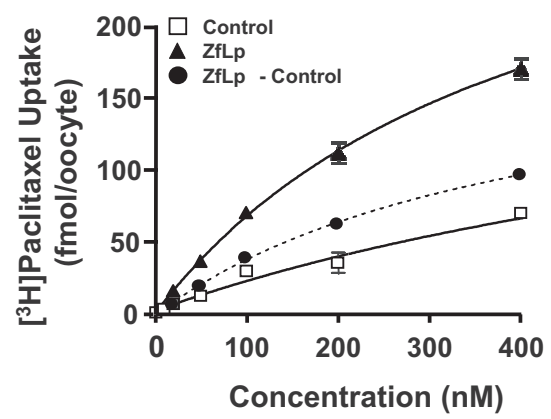

$\mathrm{D}$

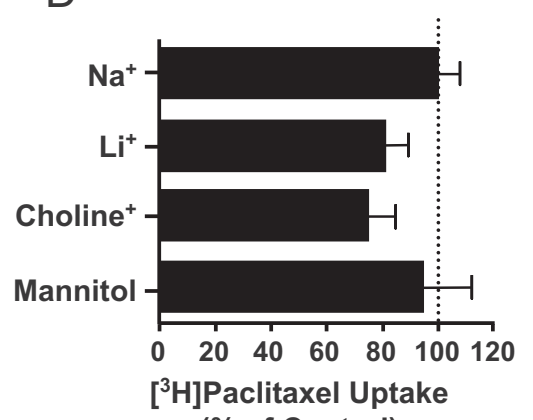

(\% of Control)

Fig. 2. Functional characterization of ZfLp in Xenopus laevis oocytes. A, Effect of $\mathrm{pH}$ on $\left[{ }^{3} \mathrm{H}\right]$ paclitaxel (taxol) transports in ZfLpexpressing oocytes. One-hour uptake of $100 \mathrm{nM}\left[{ }^{3} \mathrm{H}\right]$ paclitaxel (taxol) was measured in $\mathrm{Na}^{+}$buffers adjusted to $\mathrm{pH}$ values at 5.5 and 7.5. $\square$, un-injected; $\mathbf{\square}$, ZfLp cRNA-injected. B, Concentration-dependence of ZfLp-mediated uptake of [ $\left.{ }^{3} \mathrm{H}\right]$ paclitaxel (taxol). The uptake rate of $\left[{ }^{3} \mathrm{H}\right]$ paclitaxel (taxol) by the control and ZfLp-expresing oocytes for $1 \mathrm{hr}$ were measured at variable concentrations. $\square$, un-injected; $\boldsymbol{\Delta}$, ZfLp cRNA-injected. $\bullet$, ZfLp cRNA-injected-un-injected. C, Time-dependent uptake of [ $\left.{ }^{3} \mathrm{H}\right]$ paclitaxel (taxol) in ZfLp-expressed oocytes. The uptake of $100 \mathrm{nM}\left[{ }^{3} \mathrm{H}\right]$ paclitaxel (taxol) in oocytes expressing ZfLp was measured during $2.5 \mathrm{hr}$ of incubation. $\square$, un-injected; $\boldsymbol{\Delta}$, ZfLp cRNA-injected. $\bullet$, ZfLp cRNA-injected-uninjected. D, Effect of extracellular cation on $\left[{ }^{3} \mathrm{H}\right]$ paclitaxel (taxol) uptake by ZfLp. The uptake rates of [ $\left.{ }^{3} \mathrm{H}\right]$ paclitaxel (taxol) $(100 \mathrm{nM})$ by control oocytes or ZfLp-expressing oocytes for $1 \mathrm{hr}$ were measured in the presence or absence of extracellular $\mathrm{Na}^{+}$. Extracellular $\mathrm{Na}^{+}$was replaced with an equimolar concentration of choline, lithium, and mannitol. E, trans-Stimulatory effect of glutarate and glutathione $(\mathrm{GSH})$ on the transport of $\left[{ }^{3} \mathrm{H}\right]$ paclitaxel (taxol) via ZfLp. $100 \mathrm{nM}\left[{ }^{3} \mathrm{H}\right]$ paclitaxel (taxol) was used for the uptake experiments. Oocytes expressing ZfLp were preloaded with $10 \mu \mathrm{M}$ glutarate or $10 \mu \mathrm{M}$ GSH for $1 \mathrm{hr}$ before starting the uptake experiment. trans-Stimulatory effect was observed in the presence of glutarate and GSH. $\square$, non-injected; $\mathbf{n}$, ZfLp cRNA-injected. Data are expressed as fmol/oocyte/hr. Values are mean \pm S.E. of 12 18-oocyte determinations. The significance between control and ZfLp cRNA-injected oocytes was determined by the unpaired $t$ test $\left({ }^{*} p<\right.$ 0.01). Other experimental conditions and details are described under "Materials and Methods". GSH, glutathione. 
Isolation and pharmacological characterization of a novel gene, ZfLp

Table 1. Uptake of [ $\left.{ }^{3} \mathrm{H}\right] 5-\mathrm{FU}$ and $\left[{ }^{3} \mathrm{H}\right]$ paclitaxel (taxol) by ZfLp-expressing oocytes

\begin{tabular}{lcccc}
\hline Tracer & $\begin{array}{c}\text { Concentration } \\
(\mathrm{nM})\end{array}$ & $\begin{array}{c}\text { Non-injected } \\
(\mathrm{fmol} / \text { oocyte/hr) }\end{array}$ & $\begin{array}{c}\text { ZfLp } \\
\text { (fmol/oocyte/hr) }\end{array}$ & $\begin{array}{c}\text { Fold } \\
\text { increase }\end{array}$ \\
\hline$\left[{ }^{3} \mathrm{H}\right] 5$-FU & 100 & $36.01 \pm 4.56$ & $75.35 \pm 3.39^{*}$ & 2.1 \\
{$\left[{ }^{3} \mathrm{H}\right]$ Paclitaxel (taxol) } & 100 & $14.33 \pm 1.75$ & $61.77 \pm 2.16^{*}$ & 4.3 \\
\hline
\end{tabular}

After two days incubation, uptake experiments were performed in an ND96 solution for $1 \mathrm{hr}$. Values are mean \pm S.E. of 12-15 oocyte determinations. The significance between control (non-injected) and ZfLp-cRNA-injected oocytes was determined by the unpaired $t$ test $\left(*^{*} p<0.05\right)$.

that there was a marked difference of the expression of ZfLp mRNAs among human tissues.

To find the substrate specificity of ZfLp, the cis-inhibitory effect of various compounds by ZfLp-mediated uptake of $\left[{ }^{3} \mathrm{H}\right]$ paclitaxel (taxol) was subsequently investigated. As shown in Fig. 4, the inhibition of the ZfLpmediated transport of $\left[{ }^{3} \mathrm{H}\right]$ paclitaxel (taxol) exhibited a rank order of troleandomycin $>$ erythromycin $>$ azithromycin > clotrimazole.

Because clotrimazole exhibited the highest inhibitor for ZfLp-mediated uptake, we next studied the cis-inhibitory effect of various clotrimazole derivatives by ZfLpmediated uptake of $\left[{ }^{3} \mathrm{H}\right]$ paclitaxel (taxol). As shown in Fig. 5, inhibitory effects were observed when adding N-triphenylmethylimidazole, N-diphenylmethylimidazole and N-benzylimidazole whereas N-phenylpropylimidazole did not inhibit the transport of $\left[{ }^{3} \mathrm{H}\right]$ paclitaxel (taxol) via ZfLp, suggesting that, N-benzylimidazole would be a structural determinant for the inhibition of ZfLp-mediated drug uptake.

\section{DISCUSSION}

The ZNFs are the single largest class of transcription factors in the human genome. Previous studies have revealed that ZNFs are involved in transcriptional activation and regulation of apoptosis (Klug and Rhodes, 1987; Looman et al., 2002; Wu, 2002; Lupo et al., 2011). Alternative splicing emerges as a major mechanism of generating protein diversity. The present study describes the isolation and transport characterization of a novel gene encoding the zinc finger-like protein, ZfLp. Transport experiments using a $X$. oocyte expression system revealed that ZfLp transports $\left[{ }^{3} \mathrm{H}\right]$ paclitaxel (taxol) and $\left[{ }^{3} \mathrm{H}\right] 5-\mathrm{FU}$ in $\mathrm{pH}$-, concentration-, time-dependent and sodium-independent manners. The uptake of $\left[{ }^{3} \mathrm{H}\right]$ paclitaxel (taxol) via ZfLp was trans-stimulated by GSH and glutarate. cis-Inhibition experiment revealed that N-benzylimidazole exhibits a minimum structure for the inhibition of ZfLp-medi- ated drug transport by clotrimazole. The present paper is the first evidence concerning the transport of organic solutes via the ZNFs-related gene in the human body.

Phylogenic tree analysis revealed that the ZfLp gene belongs to the ZNF superfamily, and to ZNF415 in particular. Cheng et al. (2006) have reported that five different ZNF415 isoforms (ZNF415-1, -2, -3, -4, and -5) are expressed in adult tissues but exhibit different levels of expression by RT-PCR analysis. Their report indicates that ZNF415-5 mRNA is expressed in the liver. We examined, based on their findings, the tissue distribution and the expression of the ZfLp gene. RT-PCR analysis revealed that the ZfLp mRNA was expressed in the breast, lung, stomach, small intestine, colon and ovary in human adult tissues. However, we observed that no PCR band is detected in the liver, indicating that the expression of ZfLp is tissue-specific. Further, real-time PCR analysis revealed that the expression level of ZfLp mRNA in the small intestine and colon are low. In this respect, further study is needed to determine the reason the expression levels of ZfLp mRNA are exhibited in a differential pattern in human tissues.

With respect to the transmembrane domains (TMDs), we have reported that OSCP1/Oscp1, the lambda light chain of human immunoglobulin surface antigen-related gene (IgLC-rG), ribosomal protein L3 (RPL3) and human nucleoside transporter $1(\mathrm{hNT} 1)$ are predicted to have at least one TMD (Kobayashi et al., 2005a, 2005b, 2010a and 2010b; Umemoto et al., 2009). These transporters are predicted to be clarified as soluble proteins (http://bp.nuap.nagoya-u.ac.jp/sosui/). On the other hand, human organic solute transporters such as SLCO21A/ Slco21a, SLCO22A/Slco22a and SLC3A1/2 have several membrane-spanning domains. Similarly, ABC transporters are also predicted to have 6 to 12 membrane spanning domains (Solbach et al., 2006). One possible structural feature for activating the drug transport seems to require the transmembrane signature. We examined, therefore, the secondary model of ZfLp protein using Rose 
A

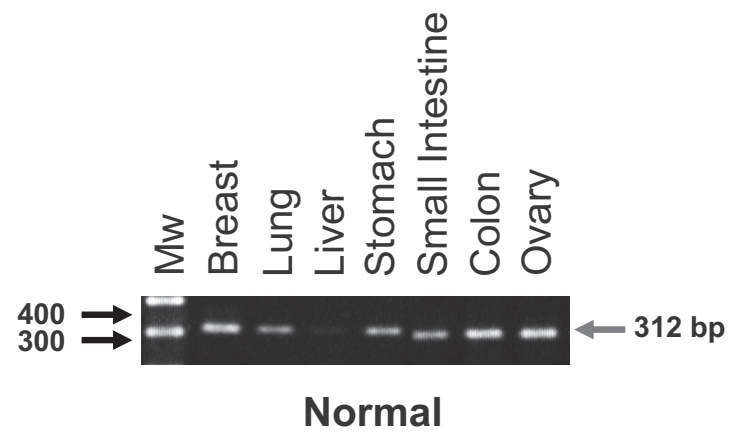

B

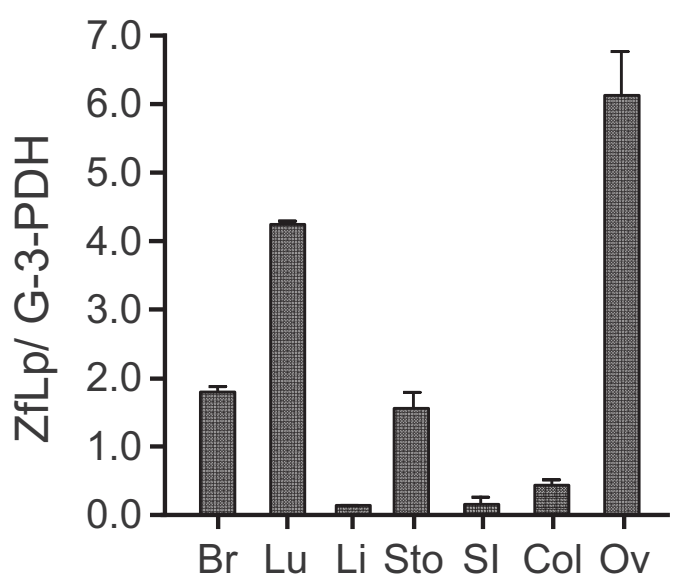

Fig. 3. Localization, distribution and expresion of ZfLp mRNA in various human tissues. A, Localization and Distribution of ZfLp mRNA by RT-PCR. The first strand cDNA prepared from human breast, lung, liver, stomach, small intestine, colon and ovary total RNA (Biochain) was used as a template for PCR amplification. The PCR products were electrophoresed on $1.0 \%$ agarose gel and visualized with ethidium bromide. The ZfLp-specific PCR product was obtained from human breast, lung, stomach, small intestine, colon and ovary. No PCR product was found in the liver. B, Expression of ZfLp mRNA by real-time PCR. The expression of the ZfLp in human various normal tissues exhibited a rank order of ovary $(\mathrm{Ov})>$ lung $(\mathrm{Lu})>$ breast $(\mathrm{Br})=$ stomach $(\mathrm{Sto})>\operatorname{colon}(\mathrm{Col})>$ small intestine $(\mathrm{SI})=$ liver $(\mathrm{Li})$.

(Window 11), Janin (Window 11), Kyte and Doolittle (Window 11), SOSUI (http://bp.nuap.nagoya-u.ac.jp/ sosui/) and TMpred analyses (http://www.ch.embnet.org/ software/TMPRED_form.html). Rose hydropathyl analy-

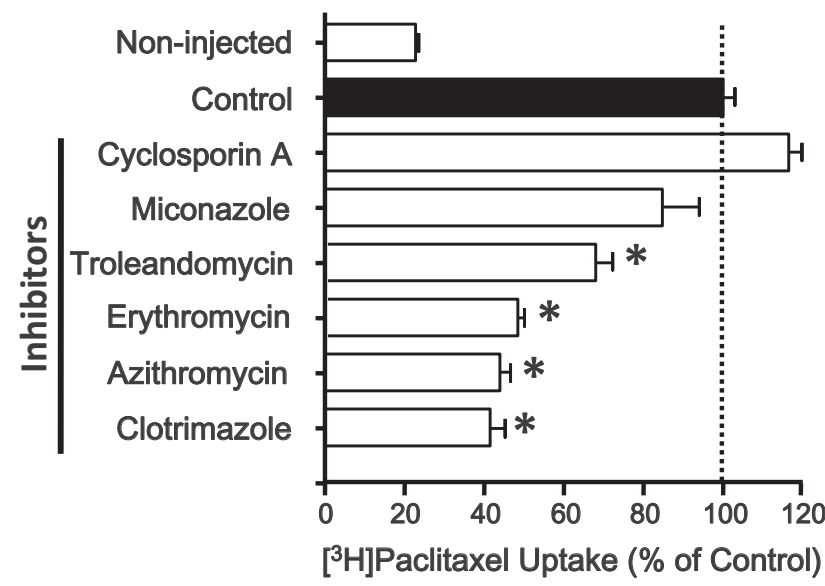

Fig. 4. cis-Inhibitory effect of ZfLp-mediated $\left[{ }^{3} \mathrm{H}\right]$ paclitaxel (taxol) uptake by various compounds. The concentration of $\left[{ }^{3} \mathrm{H}\right]$ paclitaxel (taxol) was $100 \mathrm{nM}$ and those of inhibitors in the assay medium were $10 \mu \mathrm{M}$. The values are expressed as a percentage of ZfLp-mediated $\left[{ }^{3} \mathrm{H}\right]$ paclitaxel (taxol) uptake in the absence of inhibitors. Clotrimazole exhibited a strong inhibitor for ZfLp-mediated $\left[{ }^{3} \mathrm{H}\right]$ paclitaxel (taxol) uptake. Data are expressed as the mean \pm S.E. for 18-21 oocytes.

sis predicted that there are several transmembranes; however, no membrane-spanning domains were predicted by other analyses, suggesting that, at least partly, ZfLp may have transmembrane domain(s).

Based on previous papers (Kanai et al., 1998; Ballatori et al., 2005; Kobayashi et al., 2005a, 2005b, 2010a and 2010b; Solbach et al., 2006), we conducted uptake experiments using ZfLp-cRNA-injected oocytes. Surprisingly, we found that ZfLp mediates the transport of $\left[{ }^{3} \mathrm{H}\right]$ paclitaxel (taxol) and $\left[{ }^{3} \mathrm{H}\right] 5$-FU. In addition, we observed that transport properties of $\left[{ }^{3} \mathrm{H}\right]$ paclitaxel (taxol) via ZfLp are exhibited in $\mathrm{pH}$-, concentration-, timedependent manners, and $\mathrm{Na}^{+}$-independent manner. Further, we observed that oocytes expressing ZfLp do not transport PAH and TEA (data not shown), suggesting that either anionic or cationic moieties would not be necessary for the transport recognition of ZfLp. Several possibilities regarding the ZfLp-mediated drug transport have been raised as follows; first, ZfLp could be a new transporter with an atypical structure; secondary, ZfLp could be a subunit or associated protein of a transporter which is endogenously expressed in the oocytes; thirdly, ZfLp could be an intracellular protein which influences the expression of an endogenous transporter; fourthly, overexpression of ZfLp increases or stimulates uptake of an endogenous transporter in a non-specific manner; there- 
Isolation and pharmacological characterization of a novel gene, ZfLp

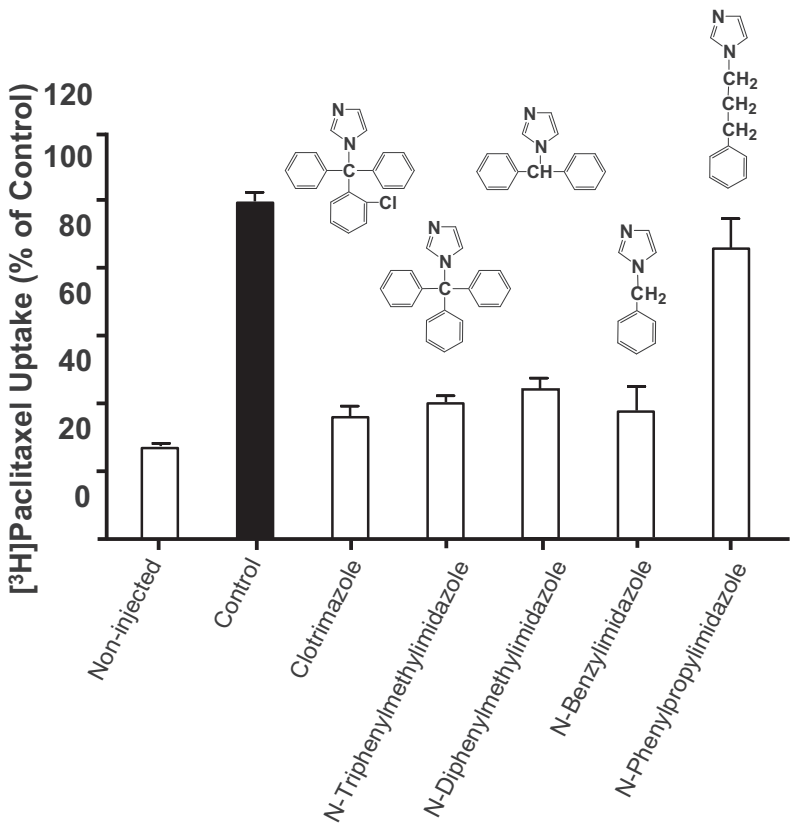

Fig. 5. cis-Inhibitory effect of ZfLp-mediated $\left[{ }^{3} \mathrm{H}\right]$ paclitaxel (taxol) uptake by clotrimazole and its structurally related derivatives. The concentration of $\left[{ }^{3} \mathrm{H}\right]$ paclitaxel (taxol) was $100 \mathrm{nM}$ and those of inhibitors in the assay medium were $10 \mu \mathrm{M}$. The values are expressed as a percentage of ZfLp-mediated $\left[{ }^{3} \mathrm{H}\right]$ paclitaxel (taxol) uptake in the absence of inhibitors. N-Benzylimidazole exhibited a minimum structure for producing the inhibition of ZfLp-mediated $\left[{ }^{3} \mathrm{H}\right]$ paclitaxel (taxol) uptake by clotrimazole. No inhibitory effect was observed when adding N-phenylpropylimidazole. Data are expressed as the mean \pm S.E. for 18-21 oocytes.

fore, further study is needed to resolve on these subject. It would be interesting to elucidate whether ZfLp is coupled to other unidentified proteins or whether ZfLp stimulates unidentified transport proteins. To distinguish whether ZfLp is a transporter/transporter or subunit/transporter associated protein, immunohistochemical analysis would be helpful to determine the subcellular localization of ZfLp.

Several endogenous compounds have been identified as the driving force of drug transporters. Sekine et al. (1997) and Sweet et al. (1997) have reported that dicarboxylates is an exchanger for stimulating rat Oat1 (rOat1[Slc22a6])mediated drug uptake. Pajor et al. (1995) have revealed that dicarboxylate also acts as the driving force of sodium-dicarboxylate cotransporter-1 (NaDC-1[Slc13a2]). Li et al. (2000) have reported that intracellular GSH is an exchange substrate and GSH stimulates the transport of organic anions via Oatp2 (Oatp1a4[Slcola4/Slc2 1a5]). On the other hand, Mahagita et al. (2007) have reported that GSH has no effect on the uptake or efflux via OATP1B1/ OATP-C/LST-1/OATP2[SLCO1B1/SLC21A6]. Recently, we have reported that cyclosporine $\mathrm{A}$ and glutathione (GSH) act as the driving force of IgLC-rG (Kobayashi et al., 2010a). Furthermore, Bahn et al. (2008) have reported that hOAT10[SLC22A13]-mediated uptake of $\left[{ }^{14} \mathrm{C}\right]$ urate is trans-stimulated by GSH. Thus, some organic solutes act as a driving force. To elucidate the driving force of ZfLp-mediated uptake, we subsequently examined GSH and dicarboxylate (glutarate) as a driving force of ZfLp. We found that a trans-stimulatory effect was observed when adding GSH and glutarate. Our results indicate that both endogenous substrates function as the driving force of ZfLp-mediated uptake and may act as an exchanger in the human body.

We subsequently studied the cis-inhibitory effect of ZfLp-mediated uptake by several organic solutes to clarify the substrate specificity. The transport of $\left[{ }^{3} \mathrm{H}\right]$ paclitaxel (taxol) via ZfLp was significantly inhibited by the addition of troleandomycin, erythromycin, azithromycin and clotrimazole whereas cyclosporine A and miconazole did not inhibit ZfLp-mediated uptake of $\left[{ }^{3} \mathrm{H}\right]$ paclitaxel (taxol). Because clotrimazole exhibited the strongest inhibitor for ZfLp-mediated [ $\left.{ }^{3} \mathrm{H}\right]$ paclitaxel (taxol) transport, we subsequently conducted the same experiment using clotrimazole and its structurally related compounds (N-triphenylmethylimidazole, N-diphenylmethylimidazole, N-benzylimidazole, and N-phenylpropylimidazole) in order to elucidate further precise substrate specificity. We found that a similar inhibitory effect was observed when adding N-triphenylmethyl-, N-diphenylmethyl-, and N-benzyl-imidazoles, whereas the transport of $\left[{ }^{3} \mathrm{H}\right]$ paclitaxel (taxol) mediated by ZfLp did not interact with $\mathrm{N}$-phenylpropylimidazole, suggesting that N-benzylimidazole would be a minimum structural unit for the inhibition of ZfLp-mediated drug uptake by clotrimazole.

There are five ZNF415 isoforms (ZNF415-1-5) known to date (Cheng et al., 2006). Phylogenic tree analysis revealed that the amino acid sequence deduced from ZfLp cDNA is positioned within a member of the ZNFs. Therefore, we assumed that ZfLp may be a novel isoform of ZNF415, presumably ZNF415-6. Cheng et al. (2006) have reported that ZNF415-1 and -5 have the highest expression in adults, while ZNF415-3 has the lowest expression level (2006). On the other hand, ZNF4152 and -4 both have a moderate level of expression. The ZNF415-1 protein is localized in the nucleus whereas the other isoforms are localized in both the nucleus and cytoplasm. Cheng et al. (2006) suggests these ZNF415 isoforms may have different roles (2006). Moreover, Eferl 
and Wagner have reported that all isoforms of ZNF415, except ZNF415-1, can inhibit AP-1 and p53 activity (Eferl and Wagner, 2003). AP-1 is a dimeric transcription factor which is mainly composed of either a Jun-Jun homodimer or a Jun-Fos heterodimer. In addition, AP-1 regulates the expression of multiple genes essential for apoptosis, differentiation, cell proliferation, cell cycle control, and tumorigenesis (Eferl and Wagner, 2003). The p53 pathway is composed of many kinds of genes and products that respond to a wide variety of stress signals. These responses to stress include apoptosis, cellular senescence or cell cycle arrest (Levine et al., 2006). Taking these facts into consideration, ZfLp may also regulate the activity of these transcription factors and may play an important role in cell cycle control or apoptosis.

In conclusion, we describe the isolation, functional characterization, and substrate selectivity of a novel human zinc finger-like protein, ZfLp. Phylogenic tree analysis suggests that isolated ZfLp is a new isoform of ZNFs, presumably ZNF415-6. Using a $X$. oocyte expression system, we discovered that ZfLp functions as a drug carrier protein. Our results may provide insights into the novel role of soluble protein such as ZNF and may contribute to the elucidation of molecular mechanism(s) of the $\left[{ }^{3} \mathrm{H}\right]$ paclitaxel (taxol) toxicity in the human body. Our results, therefore, would be expected to facilitate research on the novel role of ZNFs and on the discovery of novel drugs for targeting ZNF-related proteins such as ZfLp.

\section{ACKNOWLEDGMENTS}

This study was supported in-part-by a Private University High Technology Research Center Project matching fund subsidy from MEXT (Ministry of Education, Culture, Sports, Science and Technology).

\section{REFERENCES}

Babu, E., Takeda, M., Narikawa, S., Kobayashi, Y., Yamamoto, T., Cha, S.H., Sekine, T., Sakthisekaran, E. and Endou, H. (2002): Human organic anion transporters mediate the transport of tetracycline. Jpn. J. Pharmacol., 88, 69-76.

Bahn, A., Hagos, Y., Reuter, S., Balen, D., Brzica, H., Krick, W., Burckhardt, B.C., Sabolic, I. and Burckhardt, G. (2008): Identification of a new urate and high affinity nicotinate transporter, hOAT10 (SLC22A13). J. Biol. Chem., 283, 16332-16341.

Ballatori, N. (2005): Biology of a novel organic solute and steroid transporter, OSTalpha-OSTbeta. Exp. Biol. Med., 230, 689-698.

Bertran, J., Magagnin, S., Werner, A., Markovich, D., Biber, J., Testar, X., Zorzano, A., Kühn, L.C., Palacin, M. and Murer, H. (1992a): Stimulation of system $\mathrm{y}^{(+)}$-like amino acid transport by the heavy chain of human 4F2 surface antigen in Xenopus laevis oocytes. Proc. Natl. Acad. Sci. USA, 89, 5606-5610.

Bertran, J., Werner, A., Moore, M.L., Stange, G., Markovich, D.,
Biber, J., Testar, X., Zorzano, A., Palacin, M. and Murer, H. (1992b): Expression cloning of a cDNA from rabbit kidney cortex that indices a single transport system for cystine and dibasic and neutral amino acids. Proc. Natl. Acad. Sci. USA, 89, 56015605 .

Cha, S.H., Sekine, T., Fukushima, J., Kanai, Y., Kobayashi, Y., Goya, T. and Endou, H. (2001): Identification and characterization of human organic anion transporter 3 expressing predominantly in the kidney. Mol. Pharmacol., 59, 1277-1286.

Cha, S.H., Sekine, T., Kusuhara, H., Yu, E., Kim, J.K., Kim, D.K., Sugiyama, Y., Kanai, Y. and Endou, H. (2000): Molecular cloning and characterization of multispecific organic anion transporter 4 expressed in the placenta. J. Biol. Chem., 275, 4507-4512.

Cheng, Y., Wang, Y., Li, Y., Deng, Y., Hu, J., Mo, X., Li, N., Li, Y., Luo, N., Yuan, W., Xiao, J., Zhu, C., Wu, X. and Liu, M. (2006): A novel human gene ZNF415 with five isoforms inhibits AP-1and p53-mediated transcriptional activity. Biochem. Biophys. Res. Comm., 351, 33-39.

Eferl, R. and Wagner, E.F. (2003): AP-1: a double-edged sword in tumorigenesis. Nat. Rev. Cancer, 3, 859-868.

Hagenbuch, B. and Meier, P.J. (2004): Organic anion transporting polypeptides of the OATP/SLC21 family: Phylogenetic classification as OATP/SLCO superfamily, new nomenclature and molecular/functional properties. Pflügers Arch-Eur. J. Physiol., 447, 653-665.

Izuno, H., Kobayashi, Y., Sanada, Y., Nihei, D., Suzuki, M., Kohyama, N., Ohbayashi, M. and Yamamoto, T. (2007): Rat organic solute carrier protein 1 (rOscp1) mediated the transport of organic solutes in Xenopus laevis oocytes: Isolation and pharmacological characterization of rOscp1. Life Sci., 81, 11831192.

Kameyama, Y., Yamashita, K., Kobayashi, K., Hosokawa, M. and Chiba, K. (2005): Functional characterization of SLCO1B1 (OATP-C) variants, SLCO1B1*5, SLCO1B $1 * 15$ and SLCO1B1*15+C1007G, by using transient expression systems of HeLa and HEK293 cells. Pharmacogenet. Genomics, 15, 513522.

Kanai, Y., Segawa, H., Miyamoto, K., Uchino, H., Takeda, E. and Endou, H. (1998): Expression cloning and characterization of a transporter for large neutral amino acids activated by the heavy chain of 4F2 antigen (CD98). J. Biol. Chem., 273, 23629-23632.

Klug, A. and Rhodes, D. (1987): 'Zinc fingers': a novel protein motif for nucleic acid recognition. Trens. Biochem. Sci., 12, 464-469.

Kobayashi, Y., Matsuura, Y., Kotani, E., Fukuda, T., Aoyagi, T., Tobinaga, S., Yoshida, T. and Kuroiwa, Y. (1993): Structural requirements for the induction of hepatic microsomal cytochrome P450 by imidazole- and pyridine-containing compounds in rats. J. Biochem., 114, 697-701.

Kobayashi, Y., Ohshiro, N., Sakai, R., Ohbayashi, M., Kohyama, N. and Yamamoto, T. (2005a): Transport mechanism and substrate specificity of human organic anion transporter 2 (hOat2[SLC22A7]). J. Pharm. Pharmacol., 57, 573-578.

Kobayashi, Y., Shibusawa, A., Saito, H., Ohshiro, N., Ohbayashi, M., Kohyama, N. and Yamamoto, T. (2005b): Isolation and functional characterization of a novel organic solute carrier protein, hOSCP1. J. Biol. Chem., 280, 32332-32339.

Kobayashi, Y., Umemoto, T., Ohbayashi, M., Kohyama, N., Sanada, Y. and Yamamoto, T. (2010a): Activation of cyclosporin A transport by a novel lambda light chain of human Ig surface antigenrelated gene in Xenopus laevis oocytes. Drug Metab. Dispos., 38, 1427-1435.

Kobayashi, Y., Kawakami, K., Ohbayashi, M., Kohyama, N. and 
Isolation and pharmacological characterization of a novel gene, ZfLp

Yamamoto, T. (2010b): Ribosomal Protein L3 Mediated the Transport of Digoxin in Xenopus Laevis Oocyte. J. Toxicol. Sci., 35, 827-834.

Koepsell, H. and Endou, H. (2004): The SLC22 drug transporter family. Pflügers Arch -Eur. J. Physiol., 447, 666-676.

Lee, W.S., Rebecca, G.W., Sabbag, R.V., Mohandas, T.K. and Hediager, M.A. (1993): Cloning and chromosomal localization of a human kidney cDNA involved in cystine, dibasic, and neutral amino acid transport. J. Clin. Invest., 91, 1959-1963.

Leonard, G.D., Fojo, T. and Bates, S.E. (2003): The role of ABC transporters in clinical practice. Oncologist, 8, 411-424.

Leonessa, F. and Clarke, R. (2003): ATP binding cassette transporters and drug resistance in breast cancer. Endocr. Relat. Cancer, 10, 43-73.

Leslie, E.M., Deeley, R.G. and Cole, S.P.C. (2005): Multidrug resistance proteins: role of P-glycoprotein, MRP1, MRP2, and BCRP (ABCG2) in tissue defense. Toxicol. Appl. Pharmacol., 204, 216-237.

Levine, A.J., Hu, W. and Feng, Z. (2006): The p53 pathway: what questions remain to be explored? Cell Death Differ., 13, 10271036.

Li, L., Meier, P.J. and Ballatori, N. (2000): Oatp2 mediates bidirectional organic solute transport: a role for intracellular glutathione. Mol. Pharmacol., 58, 335-340.

Looman, C., Abrink, M., Mark, C. and Hellman, L. (2002): KRAB zinc finger proteins: an analysis of the molecular mechanisms governing their increase in numbers and complexity during evolution. Mol. Biol. Evol., 19, 2118-2130.

Lupo, A., Cesaro, E., Giorgia, M., Paola, I. and Paola, C. (2011): ZNF224: Structure and role of a multifunctional KRAB-ZFP protein. Int. J. Biochem. Cell Biol., 43, 470-473.

Maeda, K., Ieiri, I., Yasuda, K., Fujino, A., Fujikawa, H. and Otsubo, K. (2006): Effects of organic anion transporting polypeptide 1B1 haplotype on pharmacokinetics of pravastatin, valsartan, and temocapril. Clin. Pharmacol. Ther., 79, 427-439.

Mahagita, C., Grassl, S.M., Piyachaturawat, P. and Ballatori, N. (2007): Human organic anion transporter 1B1 and 1B3 function as bidirectional carriers and do not mediate GSH-bile acid cotransport. Am. J. Physiol. Gastrointest. Liver Physiol., 293, G271-G278.

Mitchell, P.J. and Tjian, R. (1989): Transcriptional regulation in mammalian cells by sequence-specific DNA binding proteins. Sciences, 245, 371-378.

Nozawa, T., Minami, H., Sugiura, S., Tsuji, A. and Tamai, I. (2005): Role of organic anion transporter OATP1B1 (OATP-C) in hepatic uptake of irinotecan and its active metabolite, 7-ethyl-10-hydroxycamptothecin: in vitro evidence and effect of single nucleotide polymorphisms. Drug Metab. Dispos., 33, 434-439.

Oswald, S., Konig, J., Lutjohann, D., Giessmann, T., Kroemer, H.K., Rimmbach, D., Rosskopf, D., Fromm, M.F. and Siegmund, W. (2008): Disposition of ezetimibe is influenced by polymorphisms of the hepatic uptake carrier OATP1B1. Pharmacogenet. Genomics, 18, 559-568.
Pajor, A.M. (1995): Sequence and functional characterization of a renal sodium/dicarboxylate cotransporter. J. Biol. Chem., 270, 5779-5785.

Rizwan, A.N. and Burckhardt, G. (2007): Organic anion transporters of the SLC22 family: biopharmaceutical, physiological, and pathological roles. Pharm. Res., 24, 450-470.

Saier, M.H.Jr. (2000): A functional-phylogenetic classification system for transmembrane solute transporters. Microbiol. Mol. Biol. Rev., 64, 354-411.

Sekine, T., Watanabe, N., Hosoyamada, M., Kanai, Y. and Endou, H. (1997): Expression cloning and characterization of a novel multispecific organic anion transporter. J. Biol. Chem., 272, 18526-18529.

Shin, H.J., Anzai, N., Enomoto, A., He, X., Kim, do K., Endou, H. and Kanai, Y. (2007): Novel liver-specific organic anion transporter OAT7 that operates the exchange of sulfate conjugates for short chain fatty acid butyrate. Hepatology, 45, 1046-1055.

Simonson, S.G., Raza, A., Martin, P.D., Mitchell, P.D., Jarcho, J.A., Brown, C.D., Windass, A.S. and Schneck, D.W. (2004): Rosuvastatin pharmacokinetics in heart transplant recipients administered an antirejection regimen including cyclosporine. Clin. Pharmacol. Ther., 76, 167-177.

Solbach, T.F., Konig, J., Fromm, M.F. and Zolk, O. (2006): ATPBinding cassette transporters in the heart. Trends Cardiovasc. Med., 16, 7-15.

Sun, W., Wu, R.R., va Poelje, P.D. and Erion, M.D. (2001): Isolation of a family of organic anion transporters from human liver and kidney. Biochem. Biophys. Res. Commun., 283, 417-422.

Sweet, D.H., Wolff, N.A. and Pritchard, J.B. (1997): Expression cloning and characterization of ROAT1. The basolateral organic anion transporter in rat kidney. J. Biol. Chem., 272, 3008830095 .

Takeda, M., Khamdang, S., Narikawa, S., Kimura, H., Kobayashi, Y., Yamamoto, T., Cha, S.H., Sekine, T. and Endou, H. (2002): Human organic anion transporters and human organic cation transporters mediate renal antiviral transport. J. Pharmacol. Exp. Ther., 300, 918-924.

Umemoto, T., Kobayashi, Y., Suzuki, M., Sanada, Y. and Yamamoto T. (2009): Cloning and pharmacological characterization of a novel gene encoding human nucleoside transporter 1 (hNT1) from a human breast cancer cDNA library. Life Sci., 84, 45-51.

Van Aubel, R.A., Masereeuw, R., Russel, F.G. (2000): Molecular pharmacology of renal organic anion transporters. Am. J. Physiol. Renal Physiol., 279, F216-F232.

Wells, R.G., Lee, W.S., Kanai, Y., Leiden, J.M. and Hediger, M.A. (1992): The 4F2 antigen heavy chain induces uptake of neutral and dibasic amino acids in Xenopus oocytes. J. Biol. Chem., 67, 15285-15288.

Wu, X. (2002): Studies on molecular mechanism of human early cardiogenesis using the Drosophila as a model system. Dev. Reprod. Biol., 11, 74-76. 